\title{
EFFECT OF IMPERFECT DIELECTRICS IN THE FIELD OF A RADIOTELEGRAPHIC ANTENNA
}

\author{
By John M. Miller, Assistant Physicist
}

\section{Measurements by Fischer ${ }^{1}$ and Austin ${ }^{2}$ have shown that the} curve which represents the variation of the resistance of an antenna with the wave length of the oscillation has two characteristic features. Starting from the wave length corresponding to the fundamental of the antenna, the equivalent resistance of the antenna rapidly decreases with increasing wave length, and reaches a minimum. As the wave length is still further increased, the resistance rises again, but in a linear manner (Fig. 2, B). The decrease in resistance is explained by a decrease in the energy radiated in the form of electromagnetic waves as the wave length increases. This so-called radiation resistance varies, as it should, inversely as the square of the wave length. It has been difficult, however, to account for the linear increase which takes place at the longer wave lengths, and it is with the explanation of this feature that the present paper is concerned.

A recent paper by Austin ${ }^{3}$ pointed out the similarity in the linear increase in resistance of an antenna at long wave lengths with the behavior of an absorbing condenser. He noticed that the resistance curve obtained for an antenna was similar to that representing the equivalent series resistance of a glass condenser ${ }^{4}$ and

1 C. Fischer, Phys. Zs., 12, p. 295; I9II.

2 L. W. Austin, J. of Wash. Acad., 1, p. 9, I9Ir; Phys. Zs., 12, p. 924, I9Ir; this Bulletin, 9, p. 65, r9I2 (Scientific Paper No. I89); Jahrb. d. drahtl. Tel., 5, p. 574; I9II-12.

3 L. W. Austin, this Bulletin, 12, p. 465, I915 (Scientific Paper No. 257); Jahrb. d. draht1. Tel., 9, p. 498, I9I5.

1 In a perfect condenser, or one which shows no energy loss, the phase of the current $(I)$ is $90^{\circ}$ in advance of the electromotive force $(E)$. In an imperfect condenser the power loss, however caused, is given by $I E$ $\sin \theta$, where $\theta$ is the phase difference or the angle by which the current lags from quadrature. An equivalent power loss is occasioned by a resistance $(\rho)$ in series with a perfect condenser when this equivalent resistance satisfies the relation $\tan \theta=C p \rho$ where $C$ is the capacity and $p=2 \pi$ times the frequency. It is characteristic of a condenser with an absorbing dielectric that the phase difference $\theta$ is, roughly, independent of the frequency, and hence the equivalent resistance must vary inversely as the frequency or directly as the wave length. 
hence concluded that dielectric absorption was a probable explanation of the phenomenon. The fact that in the curves which he had obtained for ship stations the rise in resistance was less marked than for land stations led him to believe that the absorption was probably caused by the ground acting as an imperfect dielectric. Further confirmation of this view was apparently afforded by the curve for the high-power station at Arlington, Va. This station has a very good earth connection consisting of an extensive metallic earth net just below the surface of the ground. The resistance curve for this antenna, with the steel towers earthed, showed

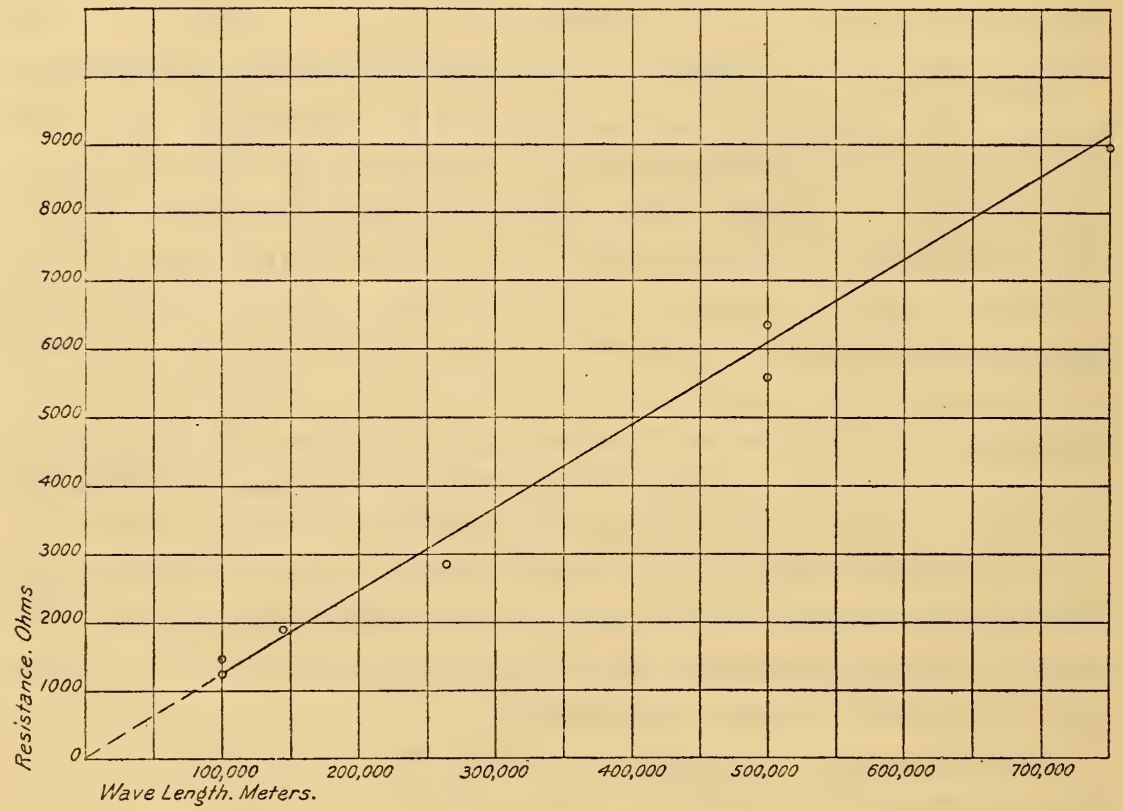

FIG. I.-Equivalent resistance of an antenna at telephone frequencies

only a slight increase in resistance for the longer waves. Austin stated that if we consider the ground as a dielectric rather than a conductor and consider it as a portion of the total dielectric lying between the antenna regarded as the upper plate of a condenser, and the ground water regarded as the lower plate, we reach a very probable explanation of many antenna resistance curves. The measurements described in this paper verify Austin's hypothesis that the effect is caused by dielectric absorption, but do not confirm the supposition that the absorbing dielectric in question is the ground. Fig. I shows the values of the equivalent resistance ob- 
tained at telephone frequencies for a small flat top antenna at the Bureau of Standards. This antenna runs from a building to a tree and has a capacity of 650 micro-microfarads (0.00065 microfarad). The measurements were made at wave lengths varying from I00 ooo to 750 ooo meters, the equivalent resistance increasing linearly from rooo to 9000 ohms. This is the order of magnitude which would be expected from Austin's measurements at wireless frequencies upon an antenna for which the rise in resistance was particularly marked.

It seemed impossible, however, to ascribe this absorption to the ground acting as an imperfect dielectric. The effect persisted at telephone frequencies while the calculations of True ${ }^{5}$ and Reich, ${ }^{6}$ based upon the measurements of conductivity and dielectric constant of the ground as given by Zenneck ${ }^{7}$ show that even for so high a frequency as would correspond to a wave length of I000 meters the magnitude of the conduction current in the ground exceeds by a hundred times that of the displacement current. The absence of absorption in the ground was also shown by measurements at telephone frequencies upon a guard plate condenser with part air and part clay between the plates. This condenser behaved as a perfect condenser with a series resistance that was independent of the frequency. Only when the clay was exceedingly dry and particularly when it was loosely packed was there any indication of absorption.

The observed large effect upon the absorption of variable air condensers brought about by the poor dielectric properties of small amounts of insulators in the electric field suggested to the author that the absorption in antennas is likewise caused by the presence of poor dielectrics in the field of the antenna. Accordingly, an experimental antenna was built in which the bad effects of poor dielectrics in the neighborhood were carefully avoided but in which any absorption that might be caused by the ground would be considerably magnified. The main capacity of the antenna consisted of six parallel wires at a distance of about 0.3 of a meter above the surface of the ground and located at a considerable distance from the nearest building or tree. The antenna was supported by four wooden posts, but was insulated from them by

${ }^{5}$ H. True, Jahrb. d. draht1. Tel., 5, p. 125; 1911-12.

${ }^{6} \mathrm{M}$. Reich. Jahrb. d. draht1. Tel., 5, pp. 176, 253; 1911-г 2.

${ }^{7}$ J. Zenneck, Ann. d. Phys., 28, p. 859; 1907. 
double porcelain insulators spaced about a meter apart. A single lead, similarly insulated, ran to the building in which the measurements were made. The earth connection was made to the water pipes of the building. The proximity of the antenna wires to the ground should reduce the lateral spread of the electrostatic field and hence the displacement through the wooden posts or other poor dielectrics, while the amount of ground between the antenna wires and ground water would be proportionately increased. The double-spaced insulators also served to reduce the

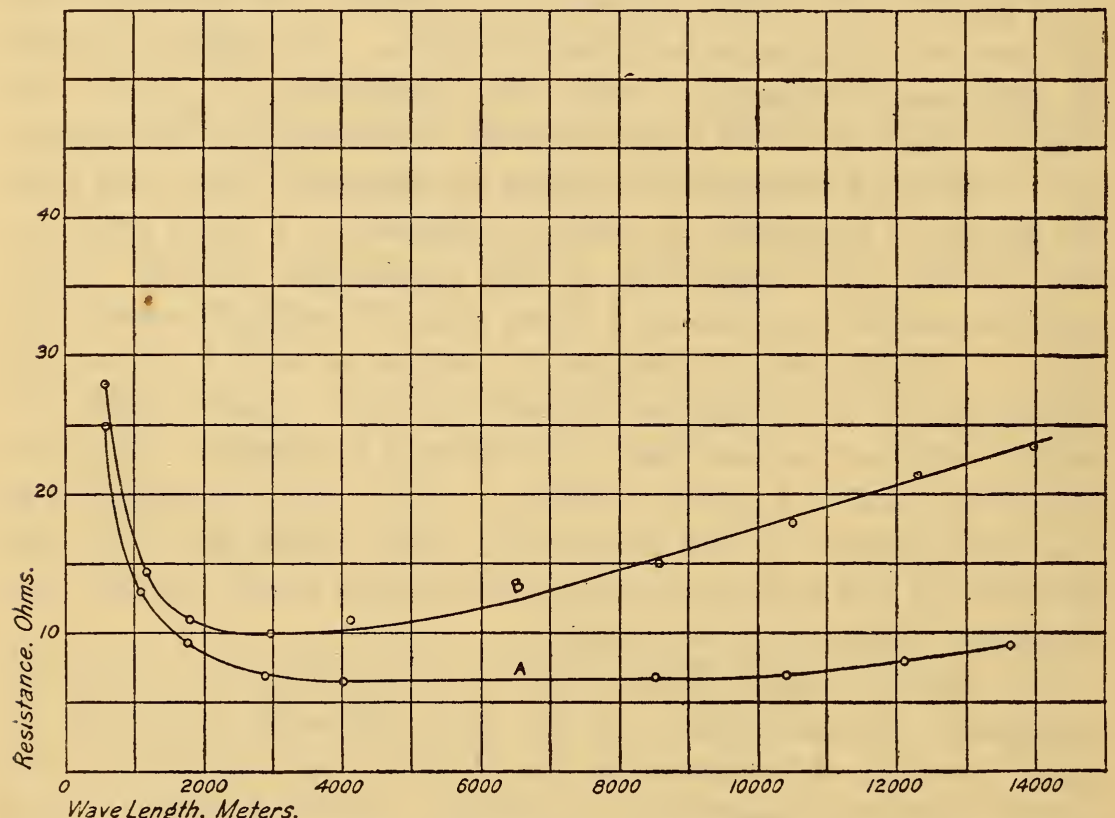

FIG. 2.- A, Resistance curve for antenna with extremely small absorption; B, effect of adding small imperfect capacity through the wooden supports

capacity through the supports. The capacity of the antenna was 850 micro-microfarads.

The resistance measurements were made by the "artificial antenna" method in which a small inductance and a condenser with a resistance in series are substituted for the antenna. The series resistance is so adjusted as to give the same current effect when the substitution is made. The resulting resistance curve, for measurements made just within the window of the building, is shown in curve $A$ of Fig. 2. The rise in resistance even at I 2000 meters is very small and probably caused by the lead wire to the 
building. The result was also verified at telephone frequencies where the absorption was barely detectable (less than 60 ohms at 3000 cycles).

Curve $B$ of the same figure shows the effect produced by adding a small capacity through the wooden supports. Wires were run from the insulated portion of the antenna to porcelain insulators on three of the stakes, the total capacity being increased by only 40 micro-microfarads or less than 5 per cent. The effect of adding this small imperfect condenser is very marked. The

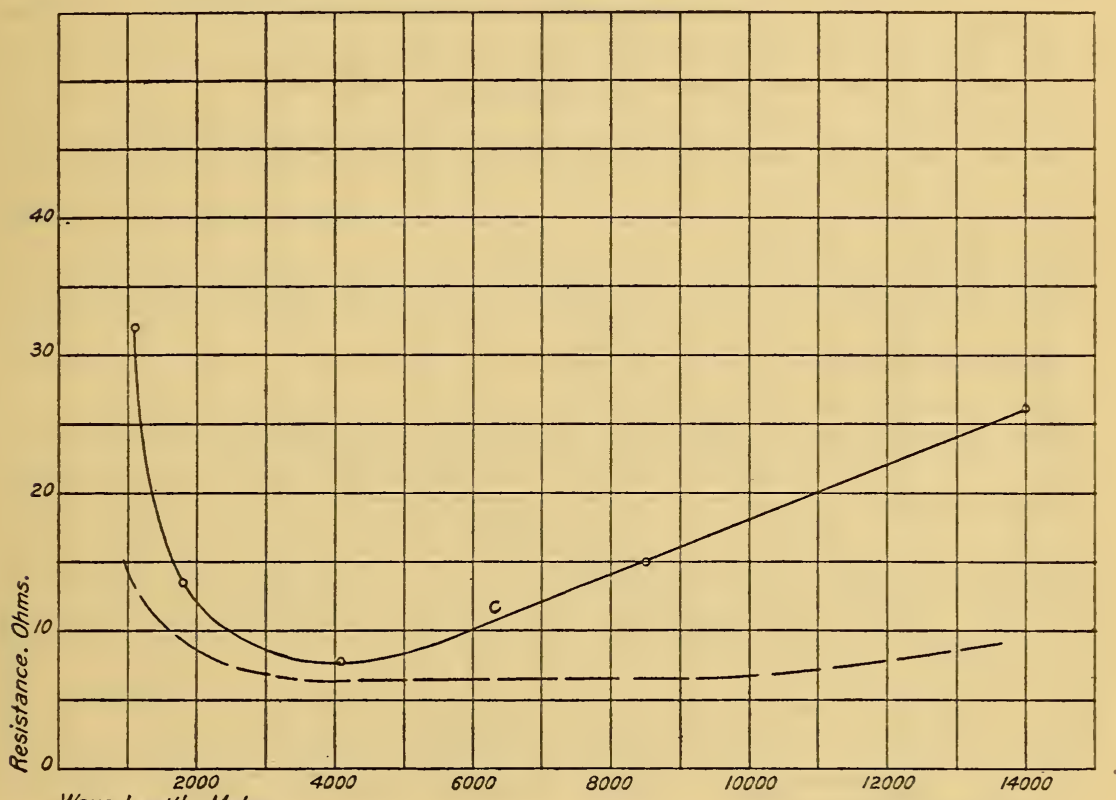

Wave Length. Meters.

FIG. 3.-C, Absorption effect produced by the portion of the leads to an antenna inside of a building

linear increase in resistance becomes pronounced, and brings with it an increase in the resistance of the antenna at all wave lengths. Austin's measurements upon ship stations, were made upon battleships, where the antennas were supported from steel masts, and it is probable that this fact may explain the slight rise of the curves obtained for such stations.

The effect of running the lead wires to an antenna inside of a building was also investigated. Curve $C$ of Fig. 3 shows the results upon the above described antenna under the same conditions as those obtaining for curve $A$ of Fig. 2 (reproduced in dash 
lines in Fig. 3), excepting that the measurements were made within the room at a distance of about 5 meters from the window. The increase in capacity in this case was 60 micromicrofarads. Curves $D$ and $E$ of Fig. 4 were obtained for antennas . completely within the building (using the same water-pipe ground as before), the former having a capacity of 290 micro-microfarads, the latter having double the capacity. In this figure the scale of resistances has been doubled. It is of interest to note that the equivalent series resistance in the case of the smaller capacity

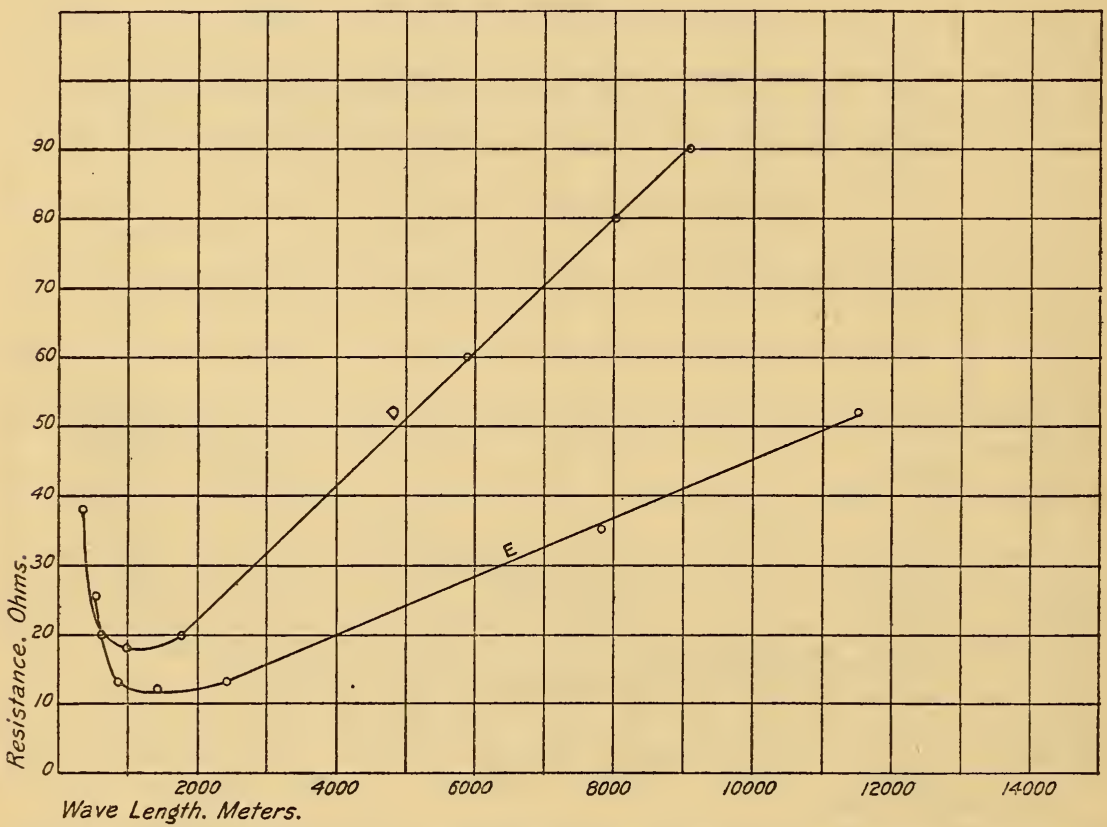

FIG. 4.-Resistance curves for antennas completely within a building. The capacity for $E$ is twice that for $D$

is approximately double that for the capacity of twice the size, which is the requisite condition for absorbing condensers with the same phase difference. Measurements, at telephone frequencies, were also made upon an antenna consisting of three wires stretched vertically along the outside of a brick building at a distance of 0.4 of a meter from the wall. The phase difference of the condenser was about 15 minutes, corresponding to an equivalent resistance of about 25 ohms at 10000 meters for a capacity of 800 micromicrofarads. The phase difference is about the same as that obtained in the case of antennas within the building. 
Finally the effect of a tree upon the absorption of an antenna was investigated. An antenna consisting of two parallel wires was strung from a building to a tree 20 meters distant at an average height of about 5 meters from the ground. The antenna terminated in a section of about 6 meters of wire which ran from limb to limb of the tree, but was insulated from it by porcelain insulators. At a distance of about 2 meters from the tree double porcelain insulators were interposed in each antenna wire, so that measurements could be made with the section in the tree included

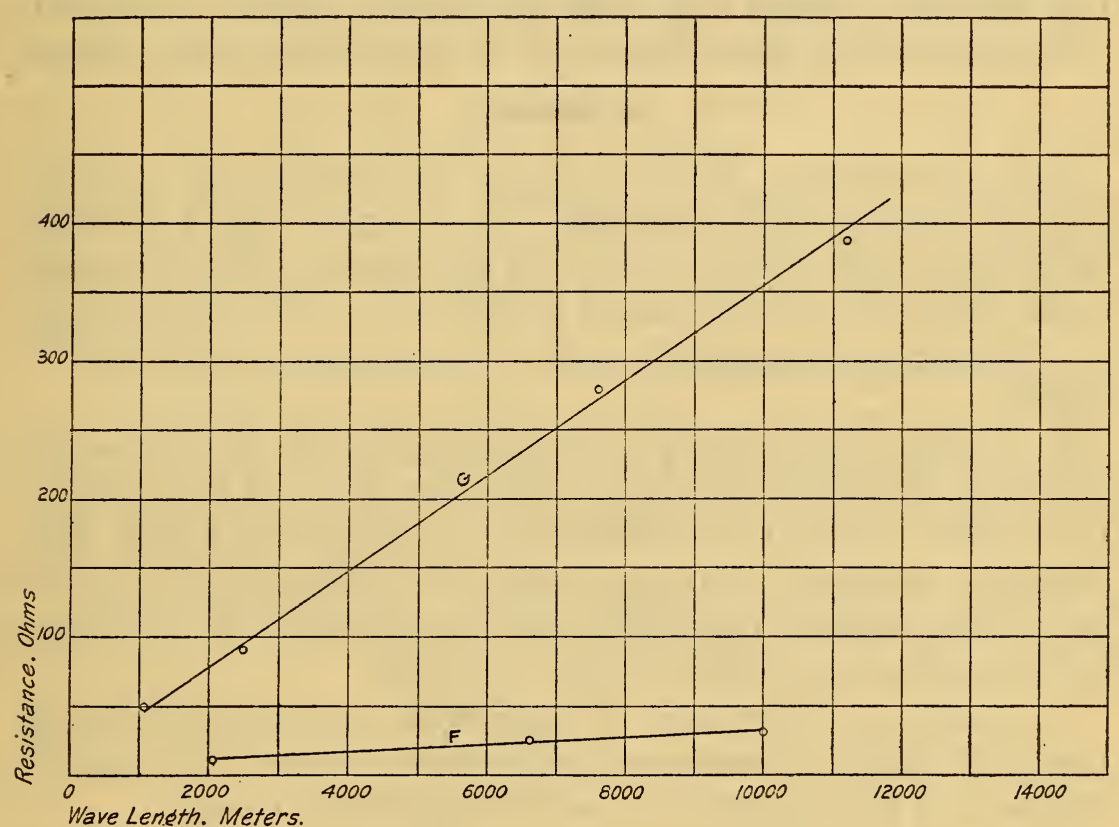

FIG. 5.-Curve $G$ shows the enormous absorption caused by a tree. Curve $F$ is the same antenna with portion in tree excluded

or excluded (using the same water-pipe ground as before). Curve $F$ of Fig. 5 was obtained for the latter case, while curve $G$ shows the enormous absorption produced by including the portion of the antenna in the tree. The capacity was increased from 390 to 540 micro-microfarads, and the phase difference of the condenser with the portion in the tree included was roughly $2^{\circ}$. The measurements were made this winter when the tree was free from foliage.

From the above it is evident that in the design of an antenna it is a matter of importance to keep the dielectric absorption of the antenna, regarded as a condenser, as low as possible in order to min- 
imize the waste of energy in the antenna and so improve its efficiency as a radiator. There is a possibility of greatly improving upon the design of existing antennas in this respect. The requirement is that the capacity through wooden masts, trees, buildings, insulators, etc., must be made extremely small in comparison to the capacity of the antenna through unobstructed air. In other words, the electrostatic field of force set up by the oscillating electric charges should not be occupied by imperfect dielectrics, and especially so where the electric force is intense. One important feature of design is to cover the interior walls of rooms into which the leads to the antenna run with grounded metal screens.

\section{SUMMARY}

The characteristic linear increase in resistance of radiotelegraphic antennas with increasing wave length (which predominates at the longer wave lengths over the decrease due to decreased radiation) is shown to be caused by dielectric absorption in imperfect dielectrics in the electric field of the antenna, as suggested by Austin.

It is shown, however, by measurements both at wireless and telephone frequencies that the absorption does not take place in the ground, but in poor dielectrics such as wooden masts, trees, buildings, insulation with poor dielectric properties, etc., in the field of the antenna, the effect being also produced when the leads to an antenna are brought within a building.

The practical importance of considering this dielectric absorption in the design of antennas is pointed out in order to increase the efficiency of the antenna as a radiator of electric energy.

In conclusion, the author desires to express his indebtedness to M. James, of this Bureau, who first suggested and carried out absorption measurements upon an antenna at telephone frequencies, and to Dr. Rosa, who was in charge of this investigation, for his many valuable suggestions during the course of the work.

WASHINGTON, January 7, I9I6. 